\title{
Lattice-based Group Enlargement for a Robot Swarm based on Crystal Growth Models
}

\author{
Kohei Yamagishi ${ }^{1}$ \\ Graduate School of Advanced Science and Technology \\ Tokyo Denki University \\ 5 Senju Asahi-cho, Adachi-ku, Tokyo 120-8551, Japan
}

\author{
Tsuyoshi Suzuki ${ }^{2}$ \\ Department of Information and communication Engineering \\ Tokyo Denki University \\ 5 Senju Asahi-cho, Adachi-ku, Tokyo 120-8551, Japan
}

\begin{abstract}
Swarm robotic systems control multiple robots in a coordinated manner for using this flexible coordination to solve complex tasks in various environments. Such systems can utilize the individual capabilities of robots scattered within the swarm as well as the collective capabilities of the assembled robots. By coordinating these capabilities, swarms can solve tasks with a range of purposes, including carrying out rough sweeps of the overall environment using scattered robots or detailed observation of a part of the environment using assembled robots. This study developed a self-organization method for constructing regular groups of robots from scattered robots to achieve coordination between individual and collective states. An approach that integrates elements of self-organization with different input information requires centralized control to manage them. To provide this self-organization without centralized control, we focus on using the phase-field method and cellular automata to facilitate crystal growth that produces ordered structures from scattered particles. We formulate a method for arranging robots in a self-organizing manner based on the geometrical regularities of tile-able lattices (honeycomb, square, and hexagonal lattices) on a two-dimensional plane, demonstrate the process undertaken in carrying out the proposed method, and quantitatively evaluate the effectiveness of the lattice-based geometrical regularity approach. The proposed method contributes to carrying out tasks with a range of purposes by organizing states with either individual or collective capabilities of robot groups.
\end{abstract}

Keywords-Multi-robot systems; self-organization; distributed control; crystal growth

\section{INTRODUCTION}

Swarm robotic systems, which apply swarm intelligence through the control of multiple homogeneous robots, have the features of scalability, flexibility, and robustness [1]. In recent years, researchers have assessed techniques for the practical application of such systems [2], [3]. Changing the manner in which the swarm is embodied can enable the robots to process multiple small tasks using parallel capabilities as well as medium- to large-scale tasks using collective capabilities. This gives the robot swarm advantages relative to single robots in carrying out large-scale/wide-area tasks such as surveillance and environment exploration and cooperative tasks such as multi-shape object transportation [4]. In this study, we examined a robot swarm-based-transporting application that manages individual and collective capabilities simultaneously to enable parallel transportation of small objects that depends on the performance of a single robot and cooperative transportation of heavy or large objects that exceeds their performances by robot groups.
When a swarm system performs multiple similar tasks, the swarm divides into multiple robot groups to carry out the tasks in parallel. However, only this simple group structure is not suitable for performing some tasks. Considering the transporting task as an example, groups with scales, shapes, and structures should be constructed such that they satisfy the size, shape, and weight of an transported object, and the system should self-organize its groups based on the given conditions.

The self-organization of a swarm robot system involves aggregation, pattern formation, and self-assembly [5]. Aggregation is a method for allocating robots to several groups from a set of scattered robots or dividing a robot swarm into several groups. Several methods have been proposed for the allocation of robots based on external factors such as task value and distance [6], [7] and the division of robot swarms based on internal factors such as the number of tasks given by the host system [8], [9]. Allocation approaches allow for the flocking of scattered robots after information relevant to the tasks has been gathered from the environment; division approaches enable robots to work in rough groups to explore an environment. Pattern formation is a method for arranging robots in pre-designed shapes. In this approach, the robot swarm will often construct designed formations from preaggregated arbitrary shapes. The robots to be added will then search for the edge of the target group or region and converge to positions suitable for enlarging the pattern designed on a 2D plane [10] or in 3D space [11], or the edge of a designed region [12]. Recently, a method focusing on reaction-diffusion systems for morphogenesis through growth was proposed as a pattern strategy [13], [14]. This approach is expected to facilitate large-scale distributed patterning because it can adapt to changing self-healing defects caused by the partial failure of the robot swarm or changes in the environmental geometry. Self-assembly is the third method for maintaining either physical or cyber positioning between organized robots. Physical connections using grippers [15], magnets [16], and welding [17] as well as virtual connections using networks and noncontact sensors [18], [19] have been proposed to facilitate selfassembly through the fabrication of rigid or elastic body-like swarms without physical constraints, respectively. In pattern formation and self-assembly, the environment-adaptive structure [14], [17] produces groups suitable for foundation shapes and structural loads that self-organize via flexible coupling by controlling the reinforcement around heavily loaded robots. By contrast, lattice-based structures [11], [18] produce groups with geometrical regularities among robots. Depending on the geometrical conditions, dense or sparse groups can be constructed, 
allowing a lattice-based structure to adapt to changes in robot density, that is, the number of robots required to carry out tasks such as coordinative transportation or observation. Thus, the implementation of self-organization requires the integration of each elemental method. This complicates the configuration of

In robot groups for transporting-which is the objective of this study-ordered arrangement enables the robots to ing their density. Self-organization through these elemental scattered robots. However, this approach requires centralized control management because the information that must be used differs by task. To solve this self-organization challenge natural phenomenon that produces lattice-based structures such as snowflakes, salt, and ores from scattered particles. This paper proposes a self-organization method for constructing tered robots without the use of centralized control. To this end, we formulate an autonomous distributed control model for introducing crystal growth into swarm robotic systems to induce enlargement.

In the process of crystal growth, particles find the surfaces of existing crystals through solidification and deposition and then adhere to positions on those crystals determined by existing meteorological conditions and molecular properties, thereby continually increasing the number of crystal layers and enlarging the crystal structure [20]. In this manner, crystal growth constitutes a self-organizational process. There are two existing mathematical tools for predicting and reproducing crystal growth: the phase-field method and cellular automata. The phase-field method describes the changes in a crystal surface during growth by calculating the state transitions of particles using scalar values that denote the stochastic state between the solid and liquid phases instead of independent thermodynamic states. Cellular automata reproduce the complex systems underpinning phenomena such as crystal growth and the formation of traffic jams. In this approach, rulesets for updating the states of cells in terms of discrete neighboring states are applied over discrete space-time intervals to reproduce macroscopic phenomena. By combining the state transitions of particles under the phase-field method with the growth rules of cellular automata, we seek to apply crystal growth to swarm robotic systems. To this end, we propose a self-organization method (Fig. 1) for constructing groups with a crystalline structure from scattered robots based on local information obtained from contact between robots.

The remainder of this paper is structured as follows.

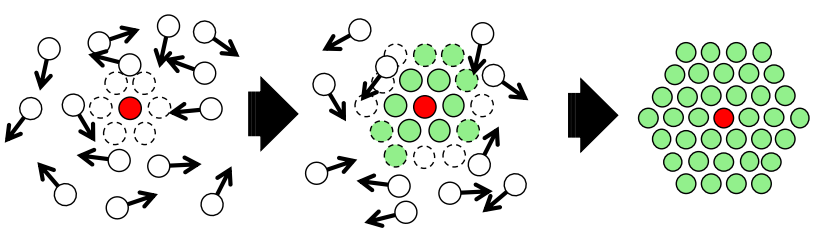

Initial scattered state Self-organization process Goal configuration robots and systems. efficiently support heavy or light objects on agerage by changtechnologies can be used to produce such an arrangement from without centralized control, we focus on crystal growth, a groups of crystalline (ordered) robot arrangements from scat-

Section II defines the configuration of robots and robot swarms and sets the problem of the self-organizing task. Sections III and IV describe, respectively, a behavioral model for robots based on state transition using the phase-field method and a layer-forming method based on cellular automata under several lattice conditions and with different coverages. In Section V, we describe the results of a robot swarm self-organization simulation based on the proposed method and quantitatively verify that the robot swarm can construct lattices with layers at arbitrary scales. Finally, Section VI concludes the paper.

\section{PRoblem StATEMENT}

In this study, we considered a swarm comprising $N$-unit homogeneous mobile robots. Each robot is equipped with a ranging sensor and local wireless communicator and can move in any direction on a two-dimensional plane within an upper velocity limit of $v[\mathrm{~m} / \mathrm{s}]$. Each mounted sensor and communicator interfaces with the sensors and communicators on other robots and can be used to observe obstacles directly on lattices tiled on the two-dimensional plane, as shown in Fig. 2. To prevent errors in measuring the distances between robots, the robots cluster into groups with circular perimeters of diameter $\sigma[\mathrm{m}]$. Under this condition, we define $r_{i j}$ as the relative distance vector between the $i$-th robot and the $j$-th neighboring robot it observes. Each robot updates its velocity control value and communicable state based on the relative distance vectors and the exchanged state for a given interval using asynchronous timing. The robots move according to the calculated velocity control values.

For constructing a group of robots to navigate various lattices from randomly arranged states on a two-dimensional plane, a landmark robot is designated to collect other robots around an observed target. The other robots find the landmark robot through environment exploration and converge on positions that enlarge the group uniformly. The resulting organized group is a regular structure based on the geometrical regularities of honeycomb, square, or hexagonal lattices that can tile a two-dimensional plane at equally spaced intervals. In this process, if the robots are not oriented so that they face in the same direction, the group cannot construct the lattice recursively; therefore, the angular references of the robots are all assumed to be aligned along the same direction.

\section{BEHAVIORAL CONTROL OF ROBOT}

To carry out the proposed self-organization process, the robots must utilize the following functionalities: environmental exploration to move individually, surface exploration to find

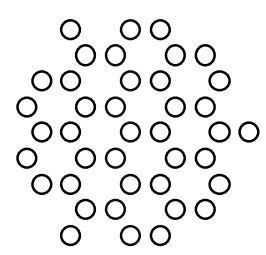

Honeycomb lattice

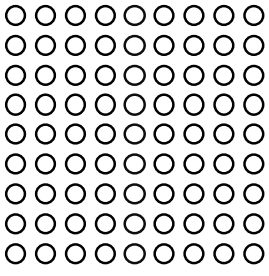

Square lattice

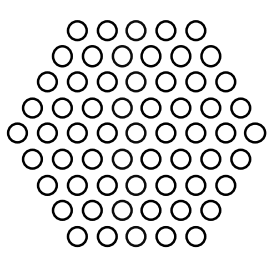

Hexagonal lattice
Fig. 1. Self-organizing a Regularly Arranged Group from Scattered Robots. 


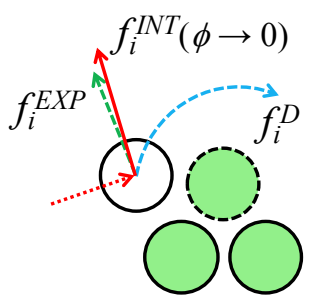

Environment exploration

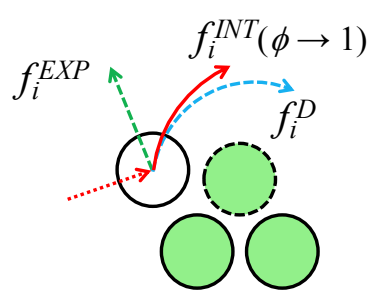

Surface exploration
Fig. 3. Illustration of Output Behavioral Vector According to the Order Parameter Value when (left) $\phi$ is Close to Zero and (right) $\phi$ is Close to One.

adherable sites on the organized group, and maintenance of the lattice. In addition, the magnitudes of these controls must be altered according to the individual robot's progress. To achieve these behavioral transitions, we apply a phase-field method to represent the state probability between the solid and liquid phases.

The phase-field method involves the application of a reaction-diffusion equation that combines diffusion based on the state difference between a particle and its neighbors with a reaction based on the particle's state to formulate the path of surface movement in crystal growth. The following simple surface movement model is used:

$$
\frac{d \phi}{d t}=\nabla^{2} \phi+8 \phi(1-\phi)(\phi-0.5+\beta)
$$

where $\phi$ is an order parameter denoting the phase of the particle, which is a continuum value between zero (liquid phase) and one (solid phase), and $\beta$ is the reaction rate, which is a constant parameter that solidifies under the condition $\beta>0$.

When a robot exchanges order parameter information with other organized robots, it updates its internal state as reflected by this equation to proceed with its solidification and transition, depending on its order parameter value, from environment exploration via object-reflecting to surface exploration via edge-following, as shown in Fig. 3. Through this solidification process, the robots exploring an environment find sites of adherence on the organized group.

Each robot recognizes other robots that have been organized based on the construction state of the lattice, $s_{j 0}$, which is obtained from the cellular automata produced by the $j$-th neighboring robot. If $s_{j 0}>0$, the neighboring robots are recognized as an organized group, with the set of recognized organized robots defined as $C S_{i}$, which is used to calculate the output of the phase-field method (in the next section, we will explain how the detailed cellular automata states are derived).

The order parameter that represents the state between the solid and liquid phases controls the $i$-th robot's behavior. When this parameter contacts the $j$-th organized robots, it is updated according to (1) as follows:

$$
\phi_{i} \leftarrow \phi_{i}+\left(\frac{\sum \phi_{j}-\phi_{i}}{\left|C S_{i}\right|}+8 \phi_{i}\left(1-\phi_{i}\right)\left(\phi_{i}-0.5+\beta\right)\right) \Delta t
$$

where $\Delta t$ is the interval of the algorithm. According to this order parameter, the controller that decides the priority of environment and surface explorations is represented as follows:

$$
f_{i}^{I N T}\left(\phi_{i}\right)=\left\{\begin{array}{ll}
\left(1-\phi_{i}\right) \hat{f}_{i}^{E X P}+\phi_{i} f_{i}^{D} & ; C S_{i} \neq \emptyset \\
\hat{f}_{i}^{E X P} & ; \text { otherwise }
\end{array} .\right.
$$

The order parameter transitions from zero to one when the crystal growth produces a solidified state. This model prioritizes surface exploration, $f_{i}^{D}$, when close to the solidification state and environment exploration, $f_{i}^{E X P}$, otherwise. Note that a robot that does not yet neighbor an organized group uses only environment exploration to find a group. These environment and surface exploration behaviors must involve interactive reflection to avoid other robots and obstacles and edge-following drift of the outline of the organized group; these behaviors are described as follows.

The avoidance behavior used in environmental exploration is generated by constructing a reflection vector between the pre-behavioral vector and the point of collision with a target as follows:

$$
f_{i}^{E X P}= \begin{cases}\sum_{j \in T S_{i}} f_{i}^{I N T}-2\left(f_{i}^{I N T} \cdot \hat{r}_{i j}\right) \hat{r}_{i j} & ; T S_{i} \neq \emptyset \\ f_{i}^{I N T} & ; \text { otherwise }\end{cases}
$$

where $T S_{i}$ is the set of reflected objects, which contains obstacles that can be contacted by the robot and neighboring robots within a balanced potential distance, $r_{0}$. To compare the magnitudes of environment and surface exploration behavior, the calculated reflection vector is normalized and integrated into the behavioral vector.

The drift behavior for surface exploration used to search for a position of adherence to an organized group is described using a vector that rotates around the neighboring robot group while maintaining the potential, i.e., the distance needed to construct the lattice. It is calculated as follows:

$$
f_{i}^{D}=f_{i}^{P}- \begin{cases}R\left(+\frac{\pi}{2}\right) \hat{r}_{i d} & ;\left|f_{i}^{I N T} \times r_{i d}\right| \geq 0 \\ R\left(-\frac{\pi}{2}\right) \hat{r}_{i d} & ;\left|f_{i}^{I N T} \times r_{i d}\right|<0\end{cases}
$$

where the first and second terms on the right-hand side are the potential functions used to maintain a constant distance between robots as an surface and the rotation surrounding the neighbor closest to the pre-behavioral vector among organized robots, $d=\forall \arg \max \left(f_{i}^{I N T} \cdot r_{i j} \mid j \in C S_{i}\right)$, respectively. The boundary conditions of the model determine the direction of this rotation along the pre-behavioral vector. By combining these, the robot can explore around an organized group at a certain distance.

The inter-robot potential works not only for drift but also for positioning. This potential generates attraction and repulsion forces that maintain the distance between robots needed to construct a group based on the lattice. As this approach focuses on a particle system, we incorporate the Lennard-Jones potential [21] as the distance potential and a simple sinusoidal potential as an angular potential, which is represented as follows: 


$$
\begin{aligned}
f_{i}^{P}=\sum_{j \in C S_{i}} \frac{1+\cos \left(L\left(\theta_{i j}+l_{i} \pi\right)\right)}{2\left|C S_{i}\right|} f^{L J}\left(r_{i j}\right) & +\sin \left(2 L \theta_{i j}\right) R\left(\frac{\pi}{2}\right) \hat{r}_{i j}
\end{aligned}
$$

where $L$ is the number of neighboring robots depending on the target lattice. $f^{L J}$ is the Lennard-Jones potential adjusted for the attraction, as shown follows:

$$
f^{L J}(r)=\frac{169 \sqrt[6]{\frac{13}{7}}}{63 \cdot 2^{\frac{5}{6}}} \frac{1}{r}\left(12\left(\frac{\sigma}{r}\right)^{12}-6\left(\frac{\sigma}{r}\right)^{6}\right) .
$$

This potential outputs a maximum attraction value of one to maintain a constant balanced distance $r_{0}$ (where $f^{L J}\left(r_{0}\right)=0$ ) that depends on the diameter of the robot. In addition to this potential, the robot is attracted in a direction that satisfies the geometric regularity of the lattice according to the period of the angular potential. By these interactions, the robot moves to the position that places the the neighbors in the direction according to the regularity of the lattice. If the robot does not maintain the lattice-based structure, such as during drift, $L$ is given zero to ensure that the angular potential does not work.

The $i$-th robot moves according to the movement vector, $v_{i}=v f_{i}^{I N T}$, based on the behavioral vector calculated using these equations.

\section{Enlarging Algorithm}

According to the behavioral model described in the previous section, a robot can find an adherence position suitable for further construction of the lattice that forms the group. We propose a ruleset of cellular automata to provide this position to the robot and a self-organizing potential function to maintain its tiled position. Fig. 4 shows the neighborhoods of these lattices, which can exchange information with neighboring robots in the same layer (unfilled layer), and the neighboring relations for constructing the lattice. The transition function of the cellular automata for these neighborhoods is represented as follows:

$$
s_{i 0} \leftarrow\left\{\begin{array}{cl}
\delta\left(s_{i 0}, s_{i 1}, s_{i 2}, s_{i 3}, s_{i 4}, s_{i 5}, s_{i 6},\right. & \\
\left.s_{i 7}, s_{i 8}, s_{i 9}, s_{i 10}, s_{i 11}, s_{i 12}\right) & ; \text { Honeycomb lattice } \\
\delta\left(s_{i 0}, s_{i 1}, s_{i 2}, s_{i 3}, s_{i 4}\right) & ; \text { Square lattice } \\
\delta\left(s_{i 0}, s_{i 1}, s_{i 2}, s_{i 3}, s_{i 4}, s_{i 5}, s_{i 6}\right) & ; \text { Hexagonal lattice }
\end{array}\right.
$$

where $s_{i 0}$ is the lattice construction state and $s_{i 1}$ to $s_{i N}$ are the neighbor states, which depend on the number of neighbors in the lattice, with $N=12,4$, and 6 denoting a honeycomb, square, and hexagonal lattice, respectively. Because each lattice has rotational symmetry around its central robot, the surrounding states can be defined by any neighboring robot [22].

To build a group, a set of transition functions, called a ruleset, that repeatedly fills and enlarges the layers of the group by counting the number of filled corners in the outer layers is applied. The construction states $s_{i 0}$ to $s_{i N}$ are therefore defined as continuous natural numbers from zero, indicating that the liquid phase searches for a position of adherence of to up to $S_{\max }$ and the solid phase fills the layers. $S_{\max }$ is at least eight, six, and eight for honeycomb, square, and hexagonal lattices, respectively, with adhered and layer-filled states included in the number of corners of the layer formed by each lattice.

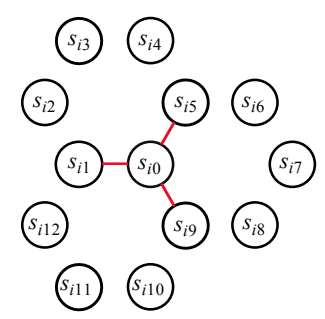

Honeycomb lattice

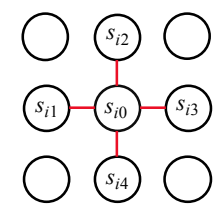

Square lattice

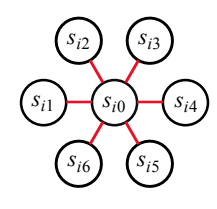

Hexagonal lattice
Fig. 4. Directly Observable Neighbors and Coupling Neighbors in Lattices.

Counting and sharing the values in the outer layers requires the neighbor values of the left and right neighbors, $s_{i l}$ and $s_{i r}$, respectively, in the layer inhabited by the $i$-th robot. The left and right reference directions of a robot can be toward either the inner or outer layers; therefore, to recognize its neighbors within its layer, the $i$-th robot calculates the layer $l_{i}$ at the current position based on the layer $l_{j}$ of the neighboring robots as $l_{i}=\min \left(l_{j} \mid j \in L S_{i}\right)+1$. For reference, the layer of the landmark robot is set to zero. $L S_{i}$, the set of neighboring robots within the range $\sqrt{2} r_{0}$, can be used to observe the robots in the inner layer from a constructible position in the outer layer. Consequently, the $i$-th robot obtains the neighbors from the layer adjacent to the inner layer as $s_{i l}$ and $s_{i r}$, respectively. The ruleset for synchronously enlarging the layers of a lattice based on these neighborhoods is then

$$
s_{i 0} \leftarrow\left\{\begin{array}{ll}
1 & ; s_{i 0}=0, \exists S_{\max } \in s_{i n} \mid 1 \leq n \leq N \\
1 & ; s_{i 0}=0,0<s_{i r}<S_{\max } \text { or } 0<s_{i l}<S_{\max } \\
s_{i r}+1 & ; 0<s_{i 0}, s_{i r}, s_{i l}<S_{\max } \text { and } \theta_{\text {ril }}<\frac{N-0.5}{2 N} \pi \\
s_{i r} & ; 0<s_{i 0}, s_{i r}, s_{i l}
\end{array} .\right.
$$

This ruleset is constructed from the top two enlarging rules and the bottom two filling rules that produce the process shown in Fig. 5. By applying these enlarging rules, robots that are adjacent to either a filled inner layer or a neighborhood in the same layer will converge to that position and be organized into the group. The robots applied this rule complete the exploration by (3) to construct the group, and apply the potential model according to the number of neighbors of the organized lattice. The robots in the outer layer then count the number of corners in that layer based on the filling rules. If the angle between $s_{i l}$ and $s_{i r}$ is less than $\pi \mathrm{rad}$, the corresponding robot is in a corner and the adjacent robots are located on the sides. The robots identified as being in corners then transition to a state that adds one to $s_{i r}$, whereas the others continue sharing $s_{i r}$. By repeating this process, the robots in the outer layer reach their maximum state, $S_{\max }$, allowing the robot swarm to synchronously enlarge the layers of the organized group.

\section{EVAluATion OF SYNCHRONiSTIC ENLARGEMENT OF RoBot AGgREGATION}

Using the proposed self-organization method, we confirmed that the self-organization process is integrated and that scattered robots can synchronously enlarge the layers of a lattice-based structure to self-organize a group. To this end, 


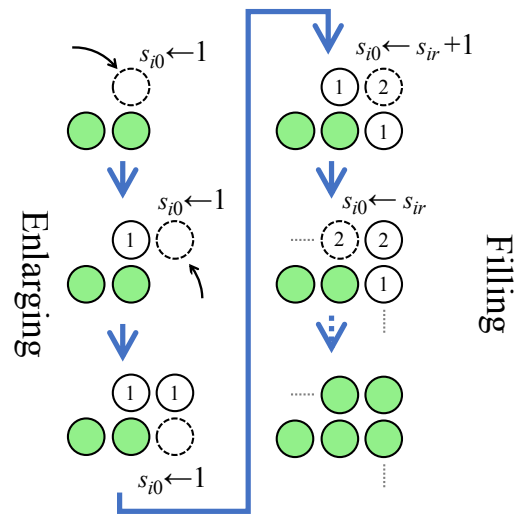

Fig. 5. Filling Process of the Robots in the Outer Layer by the Proposed Ruleset. The Robots Construct a New Layer on the Outside of the Green-colored Robots.

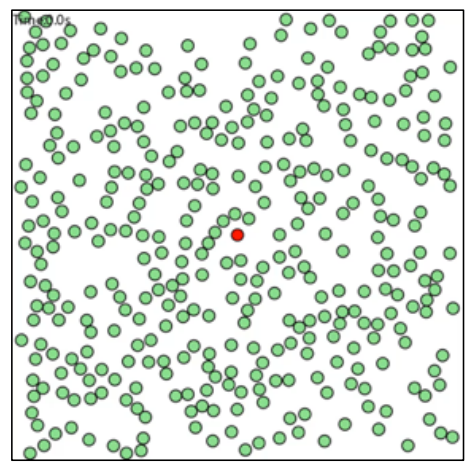

Fig. 6. Example of Field Generated by Simulation, with Green Robots Self-organizing Around a Red Landmark Robot.

we simulated the process of building self-organizing latticebased groups based on honeycomb, square, and hexagonal lattices. We also evaluated the geometrical regularities of the organized groups relative to their collective centers. Transportation requires a robot swarm comprising several tens to several hundred robots, depending on the size, weight, and shape of a transported object. In our simulation, we confirmed that the proposed method can be used to direct robots in the construction of a group to perform transporting tasks for large objects and that these groups can arrange the robots regularly to distribute the load for the weight of the transported objects.

We evaluated the dynamics of mobile robots under the following conditions: the robots were generated at random positions within a square region based on the average exploring range of an individual robot, with the landmark robot placed at the center of the region, as shown in Fig. 6. Note that this region size affects the search time of the robots. Each initialized robot had a diameter of 20 pixels, could move in all directions within an upper-velocity limit of $50 \mathrm{pixel} / \mathrm{s}$, and was able to begin moving in a random direction. The robots also updated their behavioral vectors and communicable information computed using the proposed method with a reaction coefficient $\beta$ of 0.1 at intervals of $10 \mathrm{~ms}$. We simulated the movement of the robots for each lattice and layer condition.

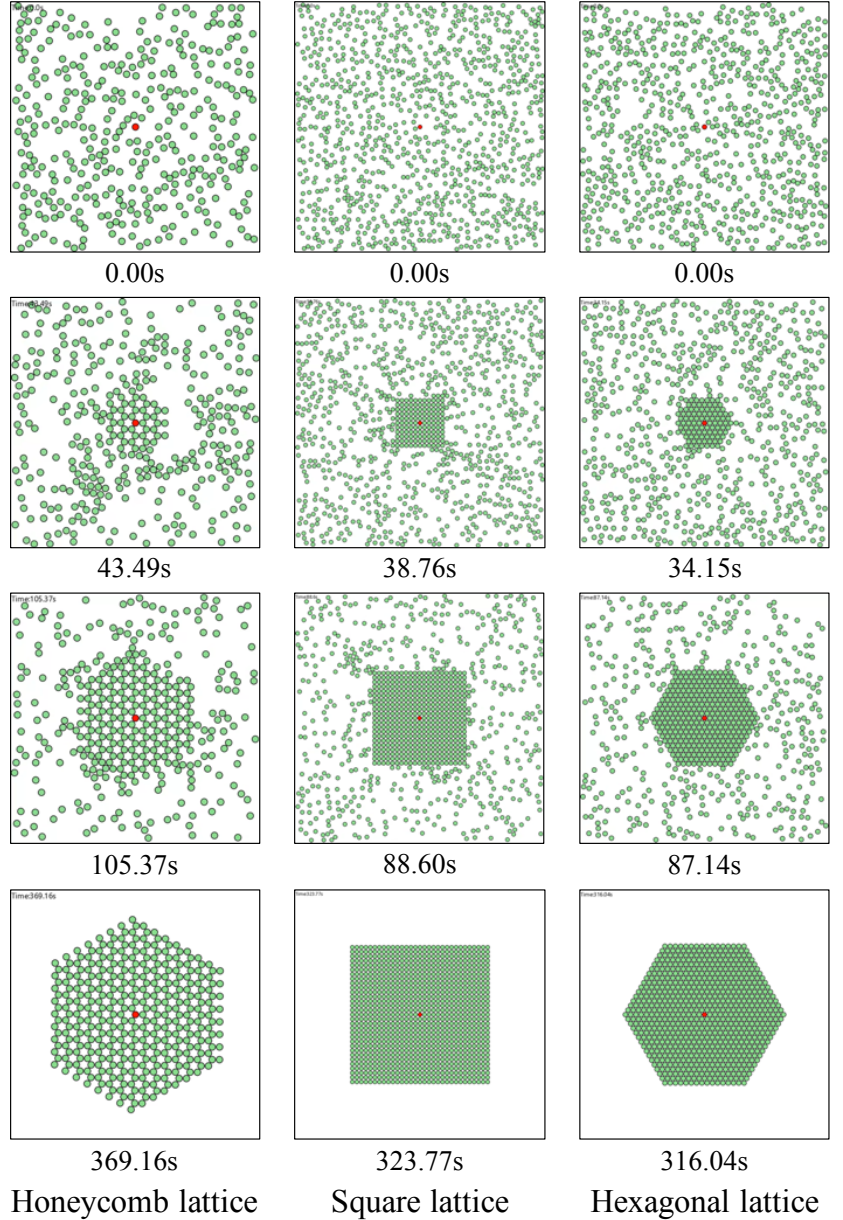

Fig. 7. Group Enlargements up to Self-organization of 15-layer Robot Groups on Different Lattice Types.

\section{A. Appearance of the Self-organization Process}

To verify that a robot swarm could synchronously enlarge a layer based on the proposed cellular automata ruleset, we simulated self-organization of 15-layer groups on honeycomb, square, and hexagonal lattices using the required number of robots $(361,481$, and 721 , respectively) for each case and setting the average exploration area to $40 \times 40$ pixel2. The selforganization results obtained under these conditions are shown in Fig. 7.

From top to bottom, the rows in the figure show the groups constructed at 0 (initial state), 5,10 , and 15 (completed state) layers, respectively. In each case, the robots, which had been initialized with individual behaviors, converged to regular positions on the applied lattice and enlarged the organized group in all directions around the landmark robot. The shapes of the in-progress and completed organized groups were, in general, similar, and there were no defects in the filled layers or overgrowth in the outermost layers of the organized groups. These results indicate that the layer-filling process based on the proposed cellular automata ruleset worked as designed, with all robot swarms applying the proposed method achieving the construction of ordered arrangements from scattered robots.

The robot behavioral paths overlap and increase depending 
on the random reflection vectors of the other robots, therefore, this paper cannot compare and discuss their time requirements. In the enlargement process, a robot exploring its environment will have to move in the path with little overlap to find an organized group. We expect to improve by incorporating behavioral models, such as random walk [23] and Lévy flight [24], that can effectively explore the overall environment into the proposed method.

\section{B. Convergence Location of Scalable Self-organization}

We then confirmed that the self-organization by a scalable robot swarm can satisfy the geometric regularity of a lattice. To evaluate this, we compared the differences between the simulated collective centers and the coordination of the landmark robots. Here, by "collective center" we mean the geometric condition-dependent coordination of the landmark robot as the organized robots converge to their ideal positions. Based on this difference, we could evaluate the geometrical regularity of the self-coordination process. The evaluation index for an individual robot is given by

$$
O_{c}=\frac{1}{100} \sum_{\text {try }=1}^{100} \sqrt{\left(\sum_{i=1}^{N} \frac{x_{i}-x_{c}}{N}\right)^{2}+\left(\sum_{i=1}^{N} \frac{y_{i}-y_{c}}{N}\right)^{2}}
$$

where $x_{c}$ and $y_{c}$ are the coordinates at which the landmark robot is initially placed, $x_{i}$ and $y_{i}$ are the coordinates of the $i$-th robot when self-organization is complete, and $N$ is the number of robots required under each experimental condition. To account for the randomness of the sequence of enlargement and the robot behavioral paths, the index was measured 100 times and the average value and standard deviation were calculated.

The index was measured for the first- to twentieth-layer group of each lattice with the average exploring area set to $60 \times 60$ pixel2. Fig. 8 shows the measurement results for each lattice. In each case, the obtained difference was shorter than the distance at which the potential model converged over time, indicating that the differences satisfied geometrical regularity. In addition, the indices for the series of honeycomb lattices were larger than those for the other lattices because, in the honeycomb lattice, the supported range of the proposed angular potential has wider than those of the others, and half of the robots in the outermost layer only construct with one robot. The indices also increased and scattered as the number of robots neighboring only one robot with weak convergence forces and wide support ranges increased with the number of layers.

\section{CONCLUSion}

This paper proposed a self-organization method that integrates the self-organization process to utilize the cooperative capability of swarm robot systems. To achieve this, we focused on crystal growth and developed a distributed control/algorithm that combines a phase state transition based on the PhaseField method and a group enlargement based on Cellular Automata. We demonstrated the self-organization process and evaluated the geometrical regularity of organized groups based on honeycomb, square, and hexagonal lattice-based arrangement conditions. From this result, we have achieved to produce

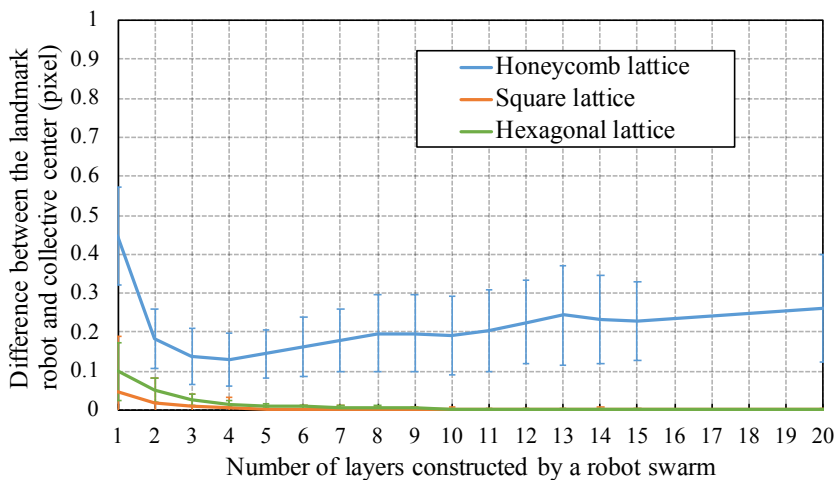

Fig. 8. Error in Measured Geometric Regularity as a Function of Number of Layers. The Solid Lines and Error Bars are the Average Values from 100 Trials and the Corresponding Standard Deviations, respectively.

a collective state from a parallel distributed state of a swarm robot system based on local robot positions and information exchange between the local robots.

Because the proposed method is a self-organizing approach based on the use of identical robots to fulfill a given lattice condition, it is limited by its inability to be used to construct flexible shapes (e.g., amorphous structures) based on longrange ordered coupling. Nevertheless, under the proposed method various group shapes can be represented by increasing the number of robots, i.e., the resolution. This will allow us to develop an approach for constructing designed group shapes by controlling the landmark robot and the layer growth speed. Furthermore, the proposed method has been shown to be effective at fixed-point observation; for the robots to engage in dynamic observation or the cooperative transportation of large objects, they will have to move in organized groups. Therefore, in the future, we will address the development of a dynamic approach in which the geometric conditions of the lattice are maintained.

\section{ACKNOWLEDGMENT}

This work was partially supported by the Research Institute for Science and Technology of Tokyo Denki University, grant number Q20D-08 / Japan. We would like to thank Editage (www.editage.com) for English language editing.

\section{REFERENCES}

[1] D. Tarapore, R. Gros, and K-P. Zauner, "Sparse Robot Swarms: Moving Swarms to Real-World Applications," Front. Robot. AI, vol. 7, no. 83, pp. 1-9, 2020, DOI: 10.3389/frobt.2020.00083.

[2] D. Carrillo-Zapata, E. Milner,J. Hird, G. Tzoumas, P.J. Vardanega, M. Sooriyabandara, M. Giuliani, A.F.T. Winfield, and S. Hauert, "Mutual Shaping in Swarm Robotics: User Studies in Fire and Rescue, Storage Organization, and Bridge Inspection," Front. Robot. AI, vol. 7, no. 53, pp.1-19, 2020, DOI: 10.3389/frobt.2020.00053.

[3] S. Batra, J. Klingner and N. Correll, "Augmented Reality for HumanSwarm Interaction in a Swarm-Robotic Chemistry Simulation," in SWARM 2021, Online, 2021, pp. 250-263.

[4] Y. Zhou, B. Rao, and W. Wang, "UAV Swarm Intelligence: Recent Advances and Future Trends," IEEE Access, vol. 8, pp. 183856-183878, 2020, DOI: 10.1109/ACCESS.2020.3028865.

[5] M. Schranz, M. Umlauft, M. Sende, and W. Elmenreich, "Swarm Robotic Behaviors and Current Applications," Front. Robot. AI, vol. 7, no. 36, pp. 1-20, 2020, DOI: 10.3389/frobt.2020.00036. 
[6] A. Jevtic, A. Gutierrez, D. Andina, and M. Jamshidi, "Distributed Bees Algorithm for Task Allocation in Swarm of Robots," IEEE Syst. J., vol. 6, no. 2, pp. 296-304, 2012, DOI: 10.1109/JSYST.2011.2167820.

[7] H. Zhao, Z. Nie, and X. Wang, "Design and Analysis of Multi-robot Grouping Aggregation Algorithm," J. Robot. Netw. Artif. Life, vol. 6, no. 1, pp. 2352-6386, 2019, DOI: 10.2991/jrnal.k.190602.002.

[8] S. Yamashita, D. Kurabayashi, and Y. Hattori, "Autonomous Division and Integration of Anonymous Agents by Using Interaction between Oscillators," Trans. JSME(in Japanese), vol. 84, no. 857, pp. 1-14, 2018, DOI: 10.1299/transjsme.17-00338.

[9] A. Barci, and C. Bettstetter, "Sandsbots: Robots That Sync and Swarm," IEEE Access, vol. 8, pp. 218752-218764, 2020, DOI: 10.1109/ACCESS.2020.3041393.

[10] M. Rubenstein, A. Cornejo, and R. Nagpal, "Programmable SelfAssembly in a Thousand-Robot Swarm," Science, vol. 345, no. 6198, pp. 795-799, 2014, DOI: 10.1126/science.1254295.

[11] Y. Zhu, D. Bie, X. Wang, Y. Zhang, H. Jin, and J. Zhao, "A Distributed and Parallel Control Mechanism for Self-Reconfiguration of Modular Robots using L-systems and Cellular Automata," J. Parallel Distrib. Comput., vol. 102, pp. 80-90, 2017, DOI: 10.1016/j.jpdc.2016.11.016.

[12] M. Alhafnawi, S. Hauert, and P. O'Dowd, "Self-Organised Saliency Detection and Representation in Robot Swarms," IEEE Robot. Autom. Lett., vol. 6, no. 2, pp. 1487-1494, 2021, DOI: 10.1109/LRA.2021.3057567.

[13] A. R. Shirazi and Y. Jin, "A Strategy for Self-Organized Coordinated Motion of a Swarm of Minimalist Robots," IEEE Trans. Emerg. Top. Comput. Intell., vol. 1, no. 5, pp. 326-338, 2017, DOI: 10.1109/TETCI.2017.2741505.

[14] D. Carrillo-Zapata, J. Sharpe, A.F.T. Winfield, L. Giuggioli, and S Hauert, "Toward Controllable Morphogenesis in Large Robot Swarms," IEEE Robot. Autom. Lett., vol. 4, no. 4, pp. 3386-3393, 2019, DOI 10.1109/LRA.2019.2926961.

[15] S. Nouyan, R. Gross, M. Bonani, F. Mondada, and M. Dorigo, "Team- work in Self-Organized Robot Colonies," IEEE Trans. Evol. Comput., vol. 13, no. 4, pp. 695-711, 2009, DOI: 10.1109/TEVC.2008.2011746.

[16] R. Zongwei and Z. Yanhe, "Self-Reconfiguration Planning of Self-Reconfigurable Robot based on Windmill-like Crystal Cell Group," in MSIE 2011, Harbin, CN, 2011, pp. 1218-1222, DOI: 10.1109/MSIE.2011.5707641.

[17] P. Swissler and M. Rubenstein, "FireAnt3D: A 3D Self-Climbing Robot Towards Non-Latticed Robotic Self-Assembly," in IROS 2020, Las Vegas, NV, USA, 2020, pp. 3340-3347.

[18] G. Lee and N.Y. Chong, "A Geometric Approach to Deploying Robot Swarms," Ann. Math. Artif. Intell., vol. 52, pp. 257-280, 2008, DOI: 10.1007/s10472-009-9125-x.

[19] H. Yang, S. Cao, L. Bai, Z. Zhang, and J. Kong, "A Distributed and Parallel Self-Assembly Approach for Swarm Robotics," Robot. Auton. Syst., vol. 118, pp. 80-92, 2019, DOI: 10.1016/j.robot.2019.04.011.

[20] A.V. Redkov, S.A. Kukushkin, and A.V. Osipov, "Spiral Growth of A Crystal Due to Chemical Reaction," J. Phys.: Conf. Ser., vol. 1124, pp.16, 2018, DOI:10.1088/1742-6596/1124/2/022006.

[21] N. Yu and A.A. Polycarpou, "Adhesive Contact Based on The Lennard-Jones Potential: A Correction to The Value of The Equilibrium Distance as Used in The Potential," J Colloid Interface Sci., vol. 278, pp.428-435, 2004, DOI:10.1016/j.jcis.2004.06.029.

[22] N.H. Packard, "Lattice Models for Solidification and Aggregation," in Science on Form, Tsukuba, JP, 1986, pp. 95-100.

[23] R.J. Alitappeh and K. Jeddisaravi, “ Multi-Robot Exploration in Task Allocation Problem," Appl Intell, 2021, DOI: 10.1007/s10489-02102483-3.

[24] Y. Katada, A. Nishiguchi, K. Moriwaki, and R. Watakabe, "Swarm Robotic Network Using Levy Flight in Target Detection Problem," Artif. Life Robot., vol. 21, pp. 295-301, 2016, DOI: 10.1007/s10015-016-02981 . 